\title{
Boundary singularity method for Stokes flow of droplet-fiber interaction
}

\author{
M. Mikhaylenko \& A. Povitsky \\ Department of Mechanical Engineering, The University of Akron, USA
}

\begin{abstract}
A method of allocation of Stokeslets and collocation points for the Boundary Singularity Method (BSM) is proposed to solve Stokes flows about single droplets and merging droplets settling on the surface of fibers in filtration process using moderate number of singularities.

In the recent studies of optimal location of singularities for Stokes flows about spherical rigid particles, the location of Stokeslets was determined as a fraction of particle radius. The current study represents further extension to the Stokeslet allocation schemes that was developed in order to evaluate Stokes flowfield about single droplets and the "peanut-shape" clusters of merging droplets settling at relatively long cylindrical fibers. Three test problems are solved to demonstrate capability of the BSM and proposed schemes of allocation of singularities. The first problem is a cross-flow about cylindrical fiber with a spherical droplet settling at its equatorial plane. This flow is modeled for a range of ratios of droplet to fiber radii. The second problem is a cross-flow about droplet on a fiber at the range of angles between flow and fiber corresponding to position of droplet on fiber ranging from attached to equatorial point of fiber to the back of fiber. The third problem is the BSM computation of Stokes force exerted on a pair of merging droplets, one of which is sliding toward another along the fiber.
\end{abstract}

Keywords: boundary singularity method, Stokes equations, microfluids, Stokeslet, allocation of Stokeslets, droplet, fiber.

\section{Introduction}

In the past decades, boundary element methods (BEM) and its variant, Boundary singularity method (BSM), have proven their efficiency in numerous fields of 
computational physics including micro- and nano- scale Stokes flows about particles, fibers and their ensembles (Zhao and Povitsky [1-4] and Zhao [5]) and became more advantageous over traditional mesh-based methods (Wrobel [6]). BEM and BSM require optimization of allocation of boundary elements to reduce the computational time and increase solution convergence. It was shown in Mikhaylenko and Povitsky [7] that CPU time of BSM method needed to obtain solution of the same accuracy in terms of Stokes force and velocity may drop 4 to 6 times depending on the scheme of allocation of singularities.

The modelling of Stokes flowfield about micro- and nano- scale droplets in proximity to fibers describes flow physics of fibrous filtration and is crucial in predicting drag force and filtration efficiency (Hosseini and Tafreshi [8], Viswanadam and Chase [9], Kulkarni et al. [10]). The modelling of flow about droplets and computation of total drag and velocities about surfaces at different stages helps to clarify particle and droplet capturing mechanism (Winkler et al. [11]). On another hand, the problems of hydrodynamic interaction between surfaces are challenging in terms of the BSM application, because singularities located near the points of contact of the bodies increase the condition number of computational matrix and negatively affect solution accuracy. Therefore, the problem of optimal allocation of singularities becomes a critical part of computational set-up.

The paper consists of the following sections. In Section 2, formulation of problem and proposed Stokeslet allocation scheme are discussed to model the system of single and merging droplets at the surface of cylindrical fiber. Section 3 represents comparison of the BSM simulation results obtained for the traditional and proposed Stokeslet allocation schemes in terms of accuracy of velocity vector field. The capability of the BSM together with the proposed Stokeslet allocation schemes to solve real practical problems is demonstrated in Section 4. Finally, the conclusions and future research objectives are presented in Section 5 .

\section{Proposed Stokeslet allocation scheme for systems of droplets and fibers}

Depending on properties of the hydrophilic or hydrophobic fiber surface, the following problems are considered in the present study. First problem considered is the Stokes flow $(R e<1)$ about spherical droplet on surface of hydrophobic cylindrical fiber (see fig. 1a)). The second problem represents the Stokes flow about merging droplets forming "peanut-shape" cluster around hydrophilic surface of cylindrical fiber (fig. 1b)). Finally, the third problem is related to hydrodynamic interaction of two droplets in the Stokes cross-flow sliding over the surface of cylindrical fiber and merging into a "peanut-shape" droplet cluster. For the third problem of the flow about two merging droplets attached to the hydrophilic fiber, the Cassinian parametric 2-D curve (Goldberg and Chen [12]) was selected to obtain a 3-D "peanut-shape" merging droplet cluster model. Similar-shape uniform Stokeslet allocation scheme presented in [7] was used to set up "peanut-shape" cluster model (fig. 1c)). 


\subsection{Formulation of the problems and solution discretization}

The governing equations to model Stokes flow are given as follows:

$$
\left\{\begin{array}{c}
\nabla \cdot \vec{u}=0 \\
0=-\nabla p+\mu \nabla^{2} \vec{u}
\end{array}\right.
$$

The equations are solved for no-slip $\left(\left.\mathrm{u}_{\theta}\right|_{\mathrm{r}=\mathrm{R}}=0\right)$ and no-penetrating $\left(\left.\mathrm{u}_{\mathrm{r}}\right|_{\mathrm{r}=\mathrm{R}}=0\right)$ boundary conditions. The droplet-fiber system is exposed to the uniform velocity cross-flow oriented in the y-direction (see fig. 1a)). No surface tension and viscoelastic deformations are considered at this study. Velocity and pressure BSM fundamental solutions in terms of sums of Stokeslets are given as follows:

$$
\begin{gathered}
u_{j}{ }^{(k)}=\frac{1}{8 \pi \mu} \sum_{i=1}^{N}\left(\frac{F_{i}{ }^{(k)}}{\left|r_{i j}\right|}+\frac{F_{i}{ }^{(m)} \cdot r_{i j}{ }^{(k)} \cdot r_{i j}{ }^{(m)}}{\left|r_{i j}\right|^{3}}\right) \\
p_{j}{ }^{(k)}=\frac{1}{4 \pi} \sum_{i=1}^{N} \frac{F_{i}{ }^{(m)} \cdot r_{i j}{ }^{3}{ }^{(m)}}{\left|\overline{r_{i j}}\right|^{3}}
\end{gathered}
$$

where $r_{i j}=\left(x_{j}-x_{i}, y_{j}-y_{i}, z_{j}-z_{i}\right)$ is a radius-vector between a Stokeslet and collocation point, indices $i$ and $j$ denote Stokeslets and collocation point respectively, $k=1,2,3$ and $m=1,2,3$ are Stokeslet, velocity and pressure vector components, $\mu$ is a dynamic viscosity. To solve the system $M F=B$, where B is the right-hand side vector of velocities at collocation points and $F$ is a vector of Stokeslet intensities, one obtains the matrix $\mathrm{M}$ applying Einstein summation rule:

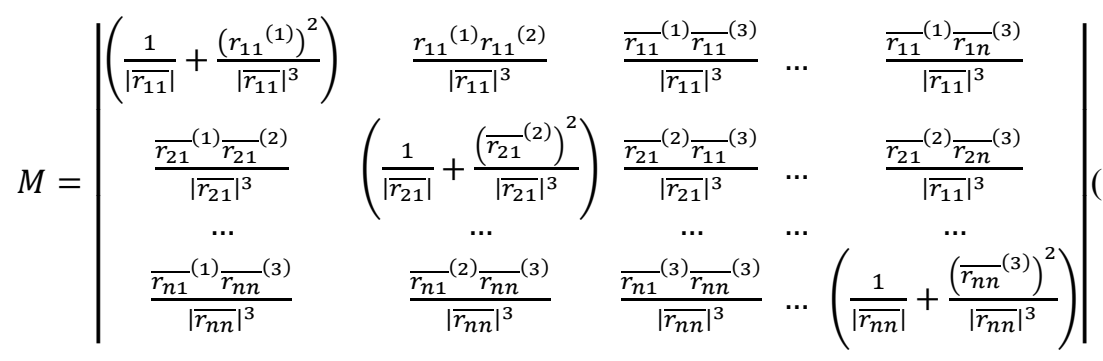

Before application of the BSM to the Stokes flow about ensembles of droplets of complex convex-concave shapes and spherical droplets in proximity to each other, a simple 3-D Stokes flow about single sphere and a 2-D Stokes flow about cylinder were solved to validate the approach. The obtained results have matched well-known analytical solutions (Fay [13], Batchelor [14]) for the sphere:

$$
F_{S S}=6 \pi \mu R U_{\infty}
$$

and for cylinder (per its unit length):

$$
F_{S C}=\frac{4 \pi \mu U_{\infty}}{\ln \left(\frac{7.4}{R e_{D}}\right)}
$$

where $U_{\infty}$ is a far-field velocity. 


\subsection{Selection of Stokeslet allocation scheme}

For the problem of the Stokes flow about spherical droplet settling on fiber depicted at fig. $1 \mathrm{a}$, the ratio of droplet to fiber $R_{\text {sphere }} / R_{\text {cylinder }}=0.5$ was selected first.

The method of submerged Stokeslets presented at fig. 1 was used, i.e. fundamental solutions were located under the surface of considered geometrical model. The normalized Stokeslet allocation radius for spherical droplet was chosen $R_{S S}=0.7$ as an optimum one proposed in [5], and the uniform Stokeslet allocation scheme was selected as described in [7].

Placement of Stokeslets for cylindrical fiber (fig. 2) was studied in terms of velocity vector field and Stokes force accuracy. In order to apply BSM to the

a)

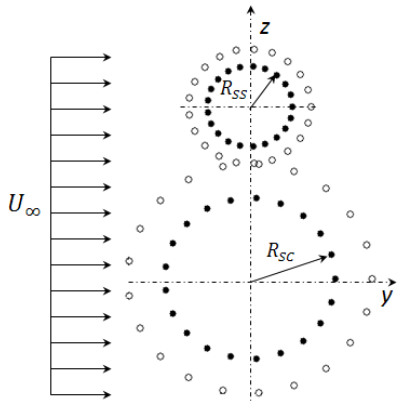

b)

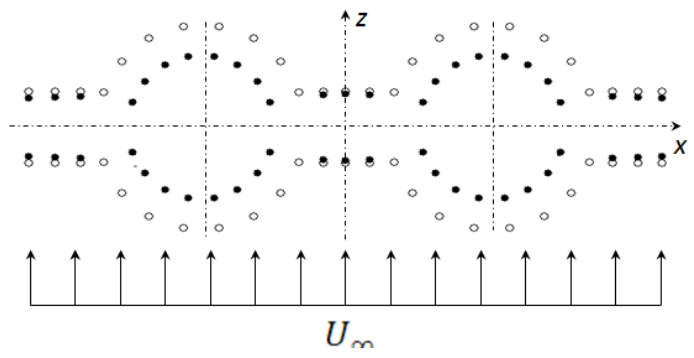

c)

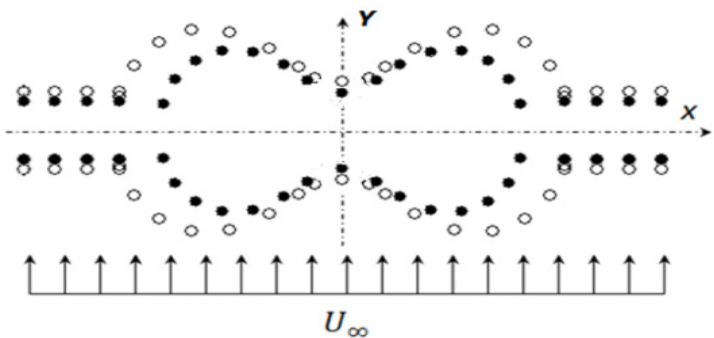

Figure 1: Problem set-up and Stokeslet allocation schemes: a) flow about droplet sticking to the fiber, b) flow about two droplets sitting on the fiber and 3) flow about droplets merging to the "peanut-shape" cluster around fiber surface. o- collocation points, $\bullet-$ Stokeslets. 
3-D Stokes flow about the finite length fiber (cylinder) and to get accurate velocity vector field, one has to place Stokeslets so as the Stokeslets' normalized allocation radius is as follows:

$$
R_{S C}=0.5 \div 0.8
$$

The analysis of geometrical setup of the Stokes flow about spherical droplet touching a fiber solved with the BSM leads to elevated condition number of the matrix M caused by near-parallel vectors directed from Stokeslets to collocation points [1-5]. This requires allocation of Stokeslets close to the surface. Values of $R_{S C}$ outside of range (6) distort velocity flow field for a cylinder and affect accuracy of the solution about sphere in proximity to the point of contact, In order to avoid this inaccuracy, the Stokeslet submergence depth in the vicinity of point of contact needs to be small enough, that is $R_{S C} \rightarrow 1$. However, numerical experiments show that as $R_{S C} \rightarrow 1$, the velocity component of the resulting flow field about cylinder is incorrect near the cylinder surface (fig. 3a)). If $R_{S C}<0.5$, the computational matrix $\mathrm{M}$ condition number increases and the solution becomes inaccurate. For traditional "equidistant" scheme with $R_{S C}=0.1$ with $N=650$ Stokeslets, the condition number increases to $1.1674 \mathrm{e}+026$. Using the same scheme with $R_{S C}=0.7$ and $N=802$ Stokeslets, the condition number obtained was $6.6079 \mathrm{e}+019$. To satisfy condition (6) apart of point of contact and to improve solution accuracy near the point of contact, the radius $R_{S C}$ should vary along the fiber depending on the distance from the point of $\operatorname{contact}(\mathrm{x}=0)$.

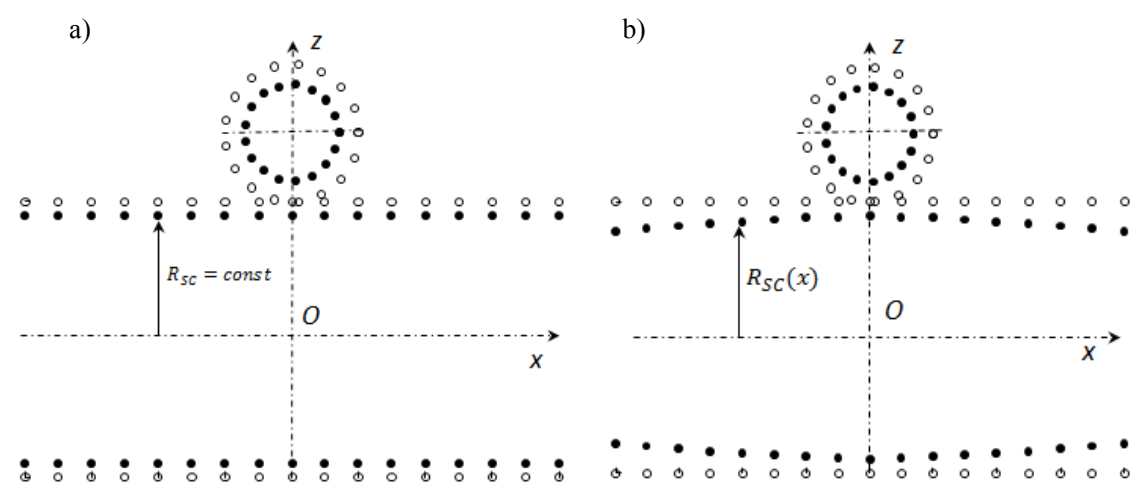

Figure 2: Stokeslet allocation scheme for the flow about droplet on a fiber: a) equidistant scheme, b) alternative scheme. $\circ$ - collocation points, -- Stokeslets.

Two schemes for 3-D flow about finite cylinder with attached droplet are considered: the traditional "equidistant" scheme (fig. 2a)), where Stokeslet placement radius $R_{S C}=$ const in the axial direction and the new scheme described here. 
The proposed scheme satisfies condition (6) apart from point of contact and, simultaneously, allows allocating Stokeslets close to surface near the contact point. The allocation depth as a function of distance to contact point is described by the following formula:

$$
R_{S C}(x)=R_{\max } \exp \left(-\frac{a|x|}{L}\right)
$$

where $x$ - axial coordinate along the cylinder, $R_{S C}$ - Stokeslet allocation radius, $R_{\max }$ - parameter defining the location of Stokeslets closest to the surface of cylinder near the point of contact with a spherical droplet at the middle length of cylinder: $x=0, a$ is a differentiation coefficient affecting the range of Stokeslet allocation radii along the fiber.

As an advantage of the proposed scheme of allocation of singularities, the $3-\mathrm{D}$ velocity vector field becomes more accurate compared to the traditional "equidistant" Stokeslet allocation scheme as shown in Section 3.

\section{Flow field for droplet attached to fiber: validation of the method}

The resulting solution for a spherical droplet touching cylindrical fiber using "equidistant" Stokeslet allocation scheme with $R_{S C}=0.97$ is shown at fig. $3 \mathrm{a}$ ). One may observe irregular velocity vectors near-penetrating the boundary of the cylinder.

For proposed Stokeslet allocation scheme applied to the case of Stokes flow about droplet on a fiber, the parameters of scheme introduced by eqn (7) were taken: $a=0.075, L=10 R$ and $R_{\max }=0.97$, where $L=10 R$ and $R=1$ are dimensionless length and radius of cylindrical fiber for this case.
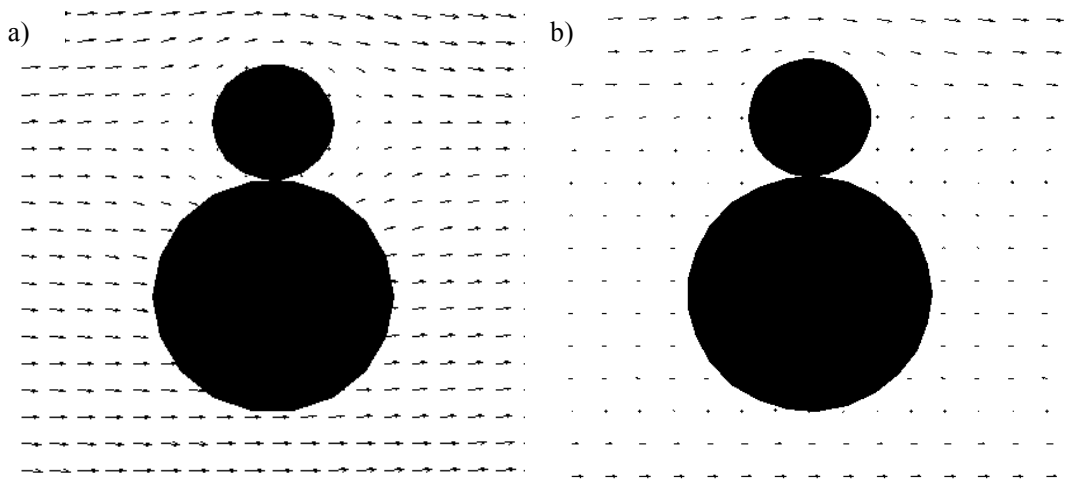

Figure 3: A ZY-plane view at 3-D velocity vector field about spherical droplet on cylindrical fiber: a) "equidistant" allocation scheme at $R_{S C}=0.97, N=677$ Stokeslets, b) proposed allocation scheme, $R_{\text {max }}=0.97, a=0.07, N=498$ (Eq. (4)) Stokeslets. 
By varying parameters $a$ and $R_{\max }$ it is possible to tune the scheme in the way to reduce the number of Stokeslets and achieve accurate flowfield parameters. For example, by changing parameter $a$ from 0.075 to 0.05 and keeping $R_{\max }=0.97$ it was possible to achieve accurate velocity vector field for $N=352$ Stokeslets instead of $N=560$ for traditional scheme. The minimum condition number achieved for proposed scheme was $1.83 \mathrm{e}+019$ for $N=444$ Stokeslets.

A similar effect in terms of velocity vector field accuracy was obtained for the problem of the Stokes flow about "peanut-shape" droplet cluster on a cylindrical fiber. Submergence of Stokeslets with $R_{S C}=0.7$ or application of proposed Stokeslet allocatiocation schemes helped resolving this issue.

\section{Application of the BSM to practical Stokes flow problems}

In order to demonstrate capability of the BSM and proposed Stokeslet allocation schemes for droplet-fiber system, three test problems of variation of the Stokes force about cylindrical fiber loaded with spherical droplets are considered as shown at fig. 1.

The first problem presumes evaluation of drag versus the radius of spherical droplet sticking to a fiber. The ratio of radii $R_{\text {sphere }} / R_{\text {cylinder }}$ varied from 0.1 to 1 (fig. 4a). The second test problem is evaluation of Stokes force at flow angles between flow velocity vector and y-axis varying from 0 to 90 degrees. The results illustrated on fig. $4 \mathrm{~b}$ were obtained for the different length-to-radius ratio for comparison. First case is presented for unit length cylinder with $R_{\text {cylinder }}=$ 1 and the second case is presented for the length-to-radius ration equal to 5 . The third problem studies the flow about two droplets, one sliding along the fiber toward another and merging into a "peanut-shape" cluster. All these problems are important in terms of determining filtering efficiency and help predicting droplets and particles capturing effects (Yarin and Chase [15]).

Stokes force (total drag force) was calculated for spherical droplet at cylindrical fiber of unit length using the proposed BSM approach and then normalized over analytical Stokes force value $F_{S C}$ for circular cylinder (5).

The results provided at Fig. $4 \mathrm{~b}$ are somewhat comparable to the total drag shown in [7] for cylindrical fiber loaded with sticking droplet dendrite for considerable solid volume fraction $\alpha$ and packing density. The solid volume fraction is determined as:

$$
\alpha=\frac{N V_{p}}{A h}
$$

where $V_{p}$ is the single droplet volume, $A$ - view area and $h$ is the depth of view. In the present test problems $\alpha=9.82 \%$, which is close to the one presented in [7]. 

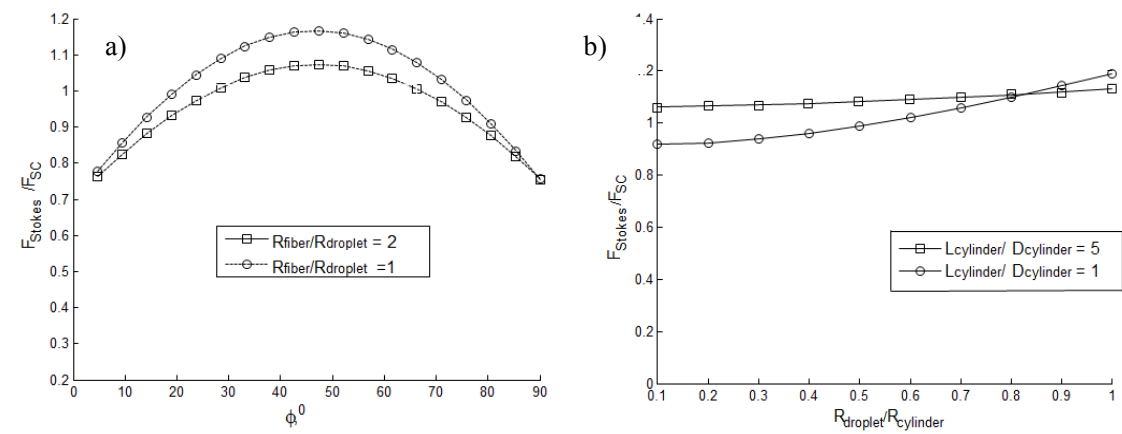

Figure 4: Stokes force (total drag) about cylindrical fiber loaded with spherical droplet: a) Drag vs. flow orientation angle, b) Total loaded fiber drag versus $R_{\text {sphere }} / R_{\text {cylinder }}$ ratio.

The Stokes flow about two droplets settling at fiber merging to "peanutshape" cluster are shown at fig. 5. The Stokes force about spherical droplets was evaluated for both longitudinal and transversal far-field flow orientation.

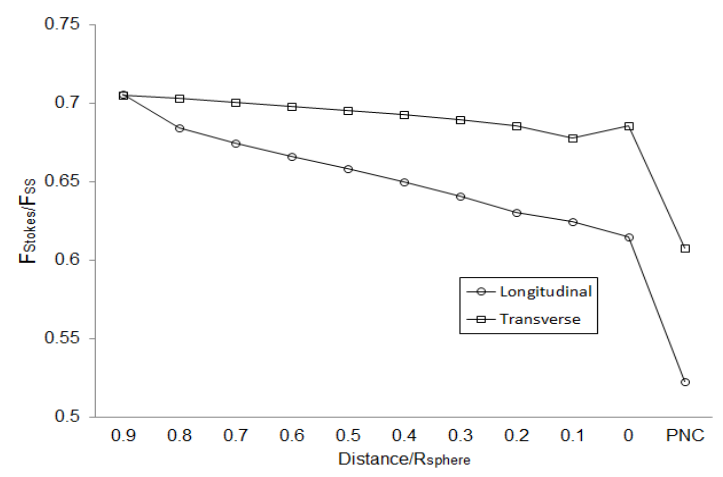

Figure 5: Stokes force (total drag) about two merging spherical droplets at cylindrical fiber versus distance between droplets normalized over droplet radius. The limiting case is a "peanut-shape" cluster (PNC) that is formed by droplets at merger phase.

It is worthwhile noting that accurate solution could not be obtained for traditional schemes (non-uniform for spherical droplet and "equidistant" for cylindrical fiber) discussed above for any Stokeslet allocation radius and any number of Stokeslets, therefore the proposed Stokeslet allocation scheme or alternative schemes are needed. 


\section{Conclusions and future work}

Simulation of the Stokes flow about droplet sticking to cylindrical fiber and about "peanut-shape" cluster of merging droplets was performed and comparison of traditional versus proposed Stokeslet allocation schemes was presented. Traditional Stokeslet allocation schemes combine non-uniform similar-shape scheme for spherical droplet and "peanut-shape" cluster and "equidistant" scheme for cylindrical fiber, while proposed scheme include "non-equidistant" (exponential) submergence depth for cylindrical fiber and uniform similar-shape scheme for spherical droplet and "peanut-shape" cluster. The large condition number of matrix $M$ represented by eqn (3) and consequent inaccuracy in solution at the point of contact and adjacent areas have been overcome with smaller Stokeslets allocation depth at those areas.

The resulting solution using BSM together with the proposed scheme of allocation of singularities shows growth of total drag with the increase of settling droplet radius. Evaluation of the results obtained for the Stokes flow about the droplet-fiber system shows that the maximum drag is achieved when the angle between the far-field flow vector and direction from the fiber axis to droplet center is 45 degrees. The results obtained for two merging droplets may be compared with the ones obtained by Goldman et al. in [16] for two particles in longitudinal or transversal translational motion in a creeping flow.

The authors plan to consider effects of surface tension of droplets interacting with solid surfaces. Another extension is toward unsteady micro- and nano- scale Stokes flow problem for both no-slip and partial-slip boundary conditions.

\section{References}

[1] Zhao, S. and Povitsky, A., Method of Fundamental Solutions for Partialslip Fibrous Filtration Flows. Int. J. Numer. Methods Fluids, 61, pp. 255274, 2009.

[2] Zhao, S. and Povitsky, A., A Hybrid Molecular and Continuum Method for Low-Reynolds-Number Flows. Nonlinear Analysis, 71, pp. 2551-2564, 2009.

[3] Zhao, S. and Povitsky, A., Method of Submerged Stokeslets for Slip Flow about Ensembles of Droplets. J. Nanosci. Nanotechnol, 8(7), pp. 37903801, 2008.

[4] Zhao, S. and Povitsky, A., Three-dimensional Boundary Singularity Method for Partial-Slip Flows. Eng. Anal. Bound. Elem, 35, pp. 114-122, 2011.

[5] Zhao, S., Development of Boundary Singularity Method for Partial-Slip and Transition Molecular-Continuum Flow Regimes with Application to Filtration. $\mathrm{PhD}$ dissertation, The University of Akron, 2009 http://etd.ohiolink.edu/view.cgi?acc_num=akron1247718304.

[6] Wrobel, L.C., The Boundary Element Method, John Wiley \& Sons Ltd., vol. 1, p. 468, 2002. 
[7] Mikhaylenko, M. and Povitsky, A., Optimal Allocation of Boundary Singularities for Stokes Flows about Clusters of Particles, Eng. Anal. Bound. Elem., paper under review, 2013.

[8] Hosseini, S.A. and Tafreshi, H. V., Modeling droplet-loaded single fiber efficiency and fiber drag using ANSYS-Fluent CFD code, Computers \& Fluids 66, pp. 157-166, 2012.

[9] Viswanadam, G. and Chase, G.G., Contact angles of drops on curved superhydrophobic surfaces, Journal of Colloid and Interface Science, 367(1), pp. 472-477, 2012.

[10] Kulkarni, P.S., Patel, S.U. and Chase, G.G., Layered Hydrophilic /Hydrophobic Fiber Media for Water-in-Oil Coalescence, Sep Pur Tech, 85, pp. 157-164, 2012.

[11] Winkler, D., Kissling, K., Schütz, S., Werfl, A. and Piesche, M., Numerical simulation of droplet-fiber-interactions in water/diesel coalescence filters, Proceedings of AFS 2010 Annual Conference and Exposition, 2010, San Antonio, TX USA.

[12] Goldberg, M.A. and Chen, C.S., The Method of Fundamental Solution for Potential and Helmholtz and Diffusion Problems In Boundary Integral Methods - Numerical and Mathematical Aspects (ed. Goldberg MA), CMP, pp. 76-103, 1998.

[13] Fay, J.A., Introduction to Fluid Mechanics, MIT Press, p. 474, 1994.

[14] Batchelor, G.K., An Introduction to Fluid Dynamics, Cambridge University Press, p. 246, 1967.

[15] Yarin, A.L. and Chase, G.G., Liquid Drop Growth on a Fiber, AIChE Journal, 52(1), pp. 217-227, 2006.

[16] Goldman, A.G., Cox, R.G. and Brenner, H., The Slow Motion of Two Identical Arbitrarily Oriented Spheres through a Viscous Fluid, Chemical Engineering Science, 21, pp. 1151-1170, 1966. 